\title{
A study of thermodynamic properties of quadratic exponential-type potential in D-dimensions
}

\author{
U.S. Okorie ${ }^{a, *}$, A.N. Ikot $^{b}$, M.C. Onyeaju ${ }^{b}$ and E.O. Chukwuocha ${ }^{b}$ \\ ${ }^{a}$ Department of Physics, Akwa Ibom State University, \\ Ikot Akpaden, P.M.B. 1167, Uyo, Nigeria. \\ *e-mail: uduakobongokorie@aksu.edu.ng \\ ${ }^{b}$ Department of Physics, Theoretical Physics Group, \\ University of Port Harcourt, Choba, Nigeria.
}

Received 13 May 2018; accepted 20 August 2018

\begin{abstract}
We solved the Schrödinger equation with Quadratic Exponential-Type Potential (QEP) model in D-dimensions using the Modified factorization method. The energy eigenvalues and total wavefunctions were obtained in a Gauss hypergeometric form. The thermodynamic properties including vibrational partition function, vibrational mean energy, vibrational mean free energy and vibrational entropy have been calculated for the electronic state of $\left(X^{1} \sum_{g}^{+}\right)$Rubidium $\left(R b_{2}\right)$ dimer. The QEP discussed can be applied extensively in Physics and Chemistry, especially in molecular dynamics.
\end{abstract}

Keywords: Quadratic exponential-type potential; modified factorization method; Schrödinger equation; vibrational partition function.

PACS: 03.65.-w; 03.65.Ge; 05.70.-a

\section{Introduction}

The importance of the exact solutions of the non-relativistic wave equations in quantum mechanics cannot be overemphasized. As such, non-relativistic spinless particles can be described using Schrödinger wave equation [1]. Most of the exactly solvable potentials are either exponential in nature. Some of these potentials that have been investigated include the Hulthen potential [2], Manning-Rosen potential [3], Eckart potential [4], Six-Parameter Exponential-Type Potential [5], Hylleraas potential [6], Eckart plus Modified Hylleraas potential [7] and other combined potentials [8]. The exact solutions of these equations with the potentials mentioned above are only possible in the s-wave where the angular momentum is zero. In a situation where the angular momentum is not zero, an approximate solution is obtained.

Different methods employed over the years in obtaining these solutions include Nikiforov-Uvarov (NU) method [912], Supersymmetry quantum mechanics (SUSYQM) [1316], Asymptotic iteration methos (AIM) [17], Proper and exact quantization rule [18-19], Factorization method [20], Functional Analysis Approach FAA (also known here as Modified Factorization Method) [21], etc. The modified factorization method is usually used to transform a secondorder homogeneous linear differential equation into a hypergeometric equation, with the help of a transformation scheme [22]. The uniqueness, simplicity and clarity of this method makes it preferable over other methods mentioned above. Recently, different authors have used this method in calculation of interaction potential energy curve and vibrational levels for Lithium molecule with the improved ex- pression of the Manning-Rosen empirical potential energy model [23]; molecular energies of the improved RosenMorse potential energy model [24]; diatomic molecule energies of the Rosen-Morse potential energy model [25]; etc.

The Quadratic exponential-type potential (QEP) model of our interest can be used in the description of molecular dynamics [26-27]. It can also be used to study the vibrational energy of diatomic molecules [28]. This potential as earlier studied [29] has various applications in Physics and Chemistry.

The thermodynamic properties for the quadratic exponential-type potential are also studied in this research. These include vibrational partition function, vibrational mean energy, vibrational mean free energy, vibrational entropy, etc. Before now, different authors have investigated the thermodynamic properties for some physical systems [30-36]. The priority of this work is fact that the thermodynamic properties of this potential have not been considered to the best of our knowledge.

The organization of this work is in different folds. Firstly, we consider the Schrödinger equation for a spherically symmetric potential in D-dimensions and solve the Schrödinger equation for its $l$-state solution. Here, we will apply an appropriate approximation scheme to the centrifugal term. The energy eigenvalues will be obtained and the corresponding wavefunction expressed in terms of hypergeometric function. The next section will be dedicated to the thermodynamic properties for the QEP model and this will be used in the study of certain mononuclear diatomic molecules. The conclusion will be given in the last section. 


\section{Solutions of the Schrödinger Equation in D- Dimensions}

The radial part of the Schrödinger equation in D-dimensions [37] is given by:

$$
\begin{aligned}
& \Psi *(r)+\frac{2 \mu}{\hbar^{2}}\left(E_{n l}-V(r)\right) \psi(r)+\frac{1}{r^{2}} \\
& \times\left[\frac{(D-1)(D-3)}{4}+l(l+D-2)\right] \psi(r)=0
\end{aligned}
$$

where $\mu$ is the reduced mass, $E_{n l}$ is the non-relativistic energy eigenvalues to be determined. The QEP model is defined as [29],

$$
V(r)=\frac{V_{0}\left(a e^{2 \alpha r}+b e^{\alpha r}+c\right)}{\left(e^{\alpha r}-1\right)^{2}}
$$

where, $a, b, c$ are the adjustable parameters of the QEP, while $V_{0}$ and $\alpha$ are the depth and the range of the QEP respectively. Substituting Eq. (2) into Eq. (1) gives:

$$
\begin{aligned}
& \Psi *(r)+\left[\frac{2 \mu E_{n l}}{\hbar^{2}}-\left(\frac{\left(a e^{2 \alpha r}+b e^{\alpha r}+c\right)}{\left(e^{\alpha r}-1\right)^{2}}+\frac{1}{r^{2}}\right.\right. \\
& \left.\left.\times\left[\frac{(D-1)(D-3)}{4}+l(l+D-2)\right]\right)\right] \psi(r)=0 .
\end{aligned}
$$

In solving Eq. (3), we invoke an approximation scheme [38] to deal with the centrifugal term since $l \neq 0$. The approximation scheme is given by:

$$
\frac{1}{r^{2}} \approx \alpha^{2}\left[\frac{1}{12}+\frac{e^{\alpha r}}{\left.e^{\alpha r}-1\right)^{2}}\right] .
$$

Substituting Eq. (4) into Eq. (3) and employing a coordinate transformation of the form $Z=\left(e^{\alpha r} / e^{\alpha r}-1\right)$, Eq. (3) reduces to:

$$
\begin{aligned}
& Z(1-Z) \psi^{\prime \prime}(Z)-(2 Z-1) \psi^{\prime}(Z)+\left[-\frac{\varepsilon_{0}}{Z(1-Z)}\right. \\
& \left.-\frac{\gamma Z}{Z(1-Z)}-\frac{\xi(1-Z)}{Z}+\Lambda\right] \psi(Z)=0 .
\end{aligned}
$$

Where

$$
\begin{gathered}
-\varepsilon_{0}=\frac{2 \mu E_{n l}}{\hbar^{2} \alpha^{2}}+\frac{1}{12}\left[\frac{(D-1)(D-3)}{4}\right. \\
+l(l+D-2)], \\
\gamma=\frac{2 \mu V_{0} a}{\hbar^{2} \alpha^{2}}, \\
\Lambda=\frac{2 \mu V_{0} b}{\hbar^{2} \alpha^{2}}-\left[\frac{(D-1)(D-3)}{4}+l(l+D-2)\right], \\
\zeta=\frac{2 \mu V_{0} c}{\hbar^{2} \alpha^{2}} .
\end{gathered}
$$

From the asymptotic behaviour of Eq. (5), (i.e., $r \rightarrow$ $0(Z \rightarrow 1)$ and $r \rightarrow \infty(Z \rightarrow 0))$, we write the wave function $\psi(Z)$ as:

$$
\psi(Z)=Z^{\nu}(1-Z)^{\omega} F(Z)
$$

Substituting Eq. (10) into Eq. (5), we obtain the following equation:

$$
\begin{aligned}
Z(1 & -Z) F^{\prime}(Z)+[1+2 \nu-(2 \nu+\omega+2) Z] F^{\prime}(Z) \\
- & {\left[(\nu+\omega)^{2}+(\nu+\omega)-(\gamma+\Lambda+\xi)\right] F(Z) } \\
+ & {\left[\frac{v^{2}-\xi-\varepsilon_{0}}{z(1-Z)}+\frac{\omega^{2}-v^{2}-\gamma+\xi}{1-z}\right] F(Z) . }
\end{aligned}
$$

Equation (11) will become a Gauss hypergeometric equation if and only the following equations vanish:

$$
\begin{aligned}
& v^{2}-\xi-\varepsilon_{0}=0 \\
& \omega^{2}-v^{2}-\gamma-\xi=0
\end{aligned}
$$

Thus, Eq. (8) now reduced to:

$$
\begin{aligned}
Z(1 & -Z) F^{\prime \prime}(Z)+[1+2 v-(2 v+2 \omega+2) Z] F^{\prime}(Z) \\
& -\left[\left(v+\omega+\frac{1}{2}+\sqrt{\frac{1}{4}+\gamma+\Lambda+\xi}\right)\right. \\
& \left.\times\left(v+\omega+\frac{1}{2}-\sqrt{\frac{1}{4}+\gamma+\Lambda+\xi}\right)\right] F(Z)=0 .
\end{aligned}
$$

From the Gauss hypergeometric function, the solution of Eq. (11) can be obtained as:

$$
\begin{aligned}
F(Z) & ={ }_{2} F_{1}\left(a_{1}, b_{1}, c_{1}, Z\right)=\frac{\Gamma\left(c_{1}\right)}{\Gamma\left(a_{1}\right) \Gamma\left(b_{1}\right)} \\
& =\sum_{k=0}^{\infty} \frac{\Gamma\left(a_{1}+k_{1}\right) \Gamma\left(b_{1}+k_{1}\right)}{\Gamma\left(c_{1}+k_{1}\right)} \frac{Z^{k_{1}}}{k_{1} !}
\end{aligned}
$$

where the unknown parameters are expressed as:

$$
\begin{aligned}
& a_{1}=v+\omega+\frac{1}{2}+\sqrt{\frac{1}{4}+\gamma+\Lambda+\xi}, \\
& b_{1}=v+\omega+\frac{1}{2}-\sqrt{\frac{1}{4}+\gamma+\Lambda+\xi} \\
& c_{1}=1+2 v
\end{aligned}
$$

Substituting Eqs. (15-18) into Eq. (10), we obtain the solution of the wave function $\psi(Z)$ as:

$$
\begin{gathered}
\psi(Z)=Z^{\nu}(1-Z)_{2}^{\omega} F_{1} V+\omega+\left(\frac{1}{2}+\sqrt{\frac{1}{4}+\gamma+\Lambda+\xi}\right. \\
\left.v+\omega+\frac{1}{2}-\sqrt{\frac{1}{4}+\gamma+\Lambda+\xi} ; 1+2 v ; Z\right)
\end{gathered}
$$


The hypergeometric function $F(Z)$ will only become a polynomial of a particular degree $(n)$ when either Eq. (16) or (17) is equal to a negative integer $(n)$. This leads to a finite hypergeometric function where $n=0,1,2,3, \ldots, n_{\max }$. Under the following quantum condition,

$$
a_{1}=-n
$$

Combining Eqs. (16), (19) and (20), we have:

$$
\begin{aligned}
& v+\omega+\frac{1}{2}+\sqrt{\frac{1}{4}+\gamma+\Lambda+\xi}=-n \\
& v+\omega+\frac{1}{2}-\sqrt{\frac{1}{4}+\gamma+\Lambda+\xi}=-n
\end{aligned}
$$

Employing Eqs. (10) and (22) respectively, results in the following expressions:

$$
\begin{aligned}
& (\omega-v)(\omega-v)=\gamma-\xi \\
& v+\omega=\sqrt{\frac{1}{4}+\gamma+\Lambda+\xi}-\frac{1}{2}-n
\end{aligned}
$$

Substituting Eq. (26) into Eq. (27) gives:

$$
\omega-v=\frac{\gamma-\xi}{\sqrt{\frac{1}{4}+\gamma+\Lambda+\xi}-\frac{1}{2}-n} .
$$

Combining Eqs. (24) and (25) appropriately, we have the following expressions given below:

$$
\begin{gathered}
v=\frac{1}{2}\left[\sqrt{\frac{1}{4}+\gamma+\Lambda+\xi}-\frac{1}{2}-n\right. \\
\left.-\frac{\gamma-\xi}{\sqrt{\frac{1}{4}+\gamma+\Lambda+\xi}-\frac{1}{2}-n}\right] \\
\omega=\frac{1}{2}\left[\sqrt{\frac{1}{4}+\gamma+\Lambda+\xi}-\frac{1}{2}-n\right. \\
\left.+\frac{\gamma-\xi}{\sqrt{\frac{1}{4}+\gamma+\Lambda+\xi}-\frac{1}{2}-n}\right],
\end{gathered}
$$

Eq. (27) above can also be written as:

$$
\omega=\left[\frac{\xi-\gamma}{2(\chi+n)}-\frac{(\chi+n)}{2}\right]
$$

Where

$$
\chi=\frac{1}{2}[1-\sqrt{4(\gamma+\Lambda+\xi)+1}]
$$

Evaluating Eq. (12) and (13) results in the expression:

$$
\varepsilon_{0}=\omega^{2}-\gamma
$$

Substituting Eqs. (6), (7) and (28) into Eq. (30) gives the non-relativistic energy eigenvalues of the Quadratic Exponential-Type Potential as:

$$
\begin{aligned}
E_{n l} & =-\frac{\hbar^{2} \alpha}{2 \mu}\left\{\left[\frac{\xi-\gamma}{2(\chi+n)}-\frac{(\chi+n)}{2}\right]^{2}\right. \\
& \left.+\frac{1}{12}\left[\frac{(D-1)(D-3)}{4}+l(l+D-2)\right]\right\}+V_{0} a
\end{aligned}
$$

Also, the corresponding total wave function in a Gauss hypergeometric form is given as:

$$
\begin{aligned}
\psi(r) & =(-1) e^{\alpha \nu r}\left(e^{\alpha \nu r}-1\right)^{-(\nu+\omega)}{ }_{2} F_{1}\left(\nu+\omega+\frac{1}{2}\right. \\
& +\sqrt{\frac{1}{4}+\gamma+\Lambda+\xi}, \nu+\omega+\frac{1}{2} \\
& \left.-\sqrt{\frac{1}{4}+\gamma+\Lambda+\xi} ; 1+2 \nu ;\left(1-e^{-\alpha r}\right)^{-1}\right)
\end{aligned}
$$

Using Eqs. (16), (14), (15) and (17), Eq. (29) can be expressed as:

$$
\begin{aligned}
\psi(r) & =(-1) e^{\alpha \nu r}\left(e^{\alpha r}-1\right)^{-(\nu+\omega)}{ }_{2} F_{1}[-n, n \\
& \left.+2\left(\nu+\omega+\frac{1}{2}\right) ; 1+2 \nu ;\left(1-e^{\alpha r}\right)^{-1}\right]
\end{aligned}
$$

\section{Partition Function and Thermodynamic Properties}

First of all, we write Eq. (31) in a closed form as:

$$
E_{n l}=-\frac{\hbar^{2} \alpha^{2}}{2 \mu}\left(\frac{P_{1}}{2(\chi+n)}-\frac{(\chi+n)}{2}\right)^{2}+P_{2}
$$

where

$$
\begin{aligned}
& P_{1}=\xi-\gamma \\
& P_{2}=V_{0} a-\frac{\hbar^{2} \alpha^{2}}{24 \mu}\left[\frac{(D-1)(D-3)}{4}+l(l+2 D-2)\right] .
\end{aligned}
$$

The partition function of the bound state system at a given temperature $T$, is given by [39],

$$
Z(\beta)=\sum_{n=0}^{\lambda} e^{-\beta E_{n l}}, \quad \beta=\frac{1}{k_{\beta} T} .
$$

Where $k_{\beta}$ is the Boltzmann constant, $E_{n l}$ is the nonrelativistic energy eigenvalues of the QEP model. Substituting Eq. (37) into Eq. (40) gives:

$$
Z(\beta)=\sum_{n=0}^{\lambda} e^{-\beta\left[P_{2}-\left(\hbar^{2} \alpha^{2} / 2 \mu\right)\left\{\left(P_{1} / 2(\chi+n)\right)-((\chi+n) / 2)\right\}^{2}\right]}
$$


Replacing the sum by an integral in the classical limit, we have:

$$
Z(\beta)=\int_{0}^{\lambda} e^{\left[L \beta \rho^{2}+\left(M / \rho^{2}\right) \beta+N \beta\right]} d \rho
$$

Due to the complicated nature of the integral contained in Eq. (39) above, we employ a Maple software to evaluate the partition function as given below [39]

$$
\begin{aligned}
Z(\beta) & =\frac{1}{2} e^{\left(L \rho^{2} \beta+N \beta\right)} \sqrt{M \beta}\left[\frac{2 \lambda e^{\left(M \beta / \lambda^{2}\right)}}{\sqrt{M \beta}}\right. \\
& \left.-\frac{2 \sqrt{M \beta} \sqrt{\pi} \operatorname{erfi}\left(\frac{\sqrt{M \beta}}{\lambda}\right)}{\sqrt{M \beta}}-2 \sqrt{\pi}\right]
\end{aligned}
$$

Where

$$
\begin{gathered}
\rho=\chi+n, \quad d \rho=d n \quad L=\frac{\hbar^{2} \alpha^{2}}{8 \mu}, \\
M=\frac{\hbar^{2} \alpha^{2} P_{1}^{2}}{8 \mu} \quad N=-\left(\frac{\hbar^{2} \alpha^{2} P_{1}}{4 \mu}+P_{2}\right)
\end{gathered}
$$

The imaginary error function given in Eq. (40) is defined as [39]:

$$
\operatorname{erfi}(x)=\frac{\operatorname{erfi}(x)}{i}=\frac{2}{\sqrt{\pi}} \int_{0}^{x} e^{t^{2}} d t
$$

Different numerical calculations can also be carried out using the imaginary error function given in Eq. (42), with the help of Maple software [40].

Using the vibrational partition function given in Eq. (40), other thermodynamic properties of the QEP model can be determined as follows:

\subsection{The Vibrational Mean Energy U}

$$
\begin{aligned}
U(\beta) & =-\frac{\partial \ln Z(\beta)}{\partial \beta}=-\frac{1}{e^{L \beta \rho^{2}+N \beta} \sqrt{M \beta}\left(\frac{2 \lambda e^{\left(M \beta / \lambda^{2}\right)}}{\sqrt{M \beta}}-2 \sqrt{\pi} \operatorname{erfi}\left(\frac{\sqrt{M \beta}}{\lambda}\right)-2 \sqrt{\pi}\right)} \\
& \times\left(2 \left(\frac{1}{2}\left(L \rho^{2}+N\right) e^{L \beta \rho^{2}+N \beta} \sqrt{M \beta}\left(\frac{2 \lambda e^{\left(M \beta / \lambda^{2}\right)}}{\sqrt{M \beta}}-2 \sqrt{\pi} \operatorname{erfi}\left(\frac{\sqrt{M \beta}}{\lambda}\right)-2 \sqrt{\pi}\right)\right.\right. \\
& \left.\left.+\frac{1}{4} \frac{e^{L \beta \rho^{2}+N \beta}\left(\frac{2 \lambda e^{\left(M \beta / \lambda^{2}\right)}}{\sqrt{M \beta}}-2 \sqrt{\pi} e r f i\left(\frac{\sqrt{M \beta}}{\lambda}\right)-2 \sqrt{\pi}\right) M}{\sqrt{M \beta}}-\frac{1}{2} \frac{e^{L \beta \rho^{2}+N \beta} \lambda e^{\left(M \beta / \lambda^{2}\right)}}{\beta}\right)\right)
\end{aligned}
$$

\subsection{Vibratioal Mean Free Energy, F}

$$
F(\beta)=-\beta^{-1} \ln Z(\beta)=-\beta^{-1} \ln \left(\frac{1}{2} e^{\left(L \rho^{2} \beta+N \beta\right)} \sqrt{M \beta}\left[\frac{2 \lambda e^{\left(M \beta / \lambda^{2}\right)}}{\sqrt{M \beta}}-\frac{2 \sqrt{M \beta} \sqrt{\pi} \operatorname{erfi}\left(\frac{\sqrt{M \beta}}{\lambda}\right)}{\sqrt{M \beta}}-2 \sqrt{\pi}\right]\right)
$$

\subsection{Vibrational Entropy, S}

$$
\begin{aligned}
S(\beta)= & k \ln Z(\beta)-k \beta \frac{\partial \ln Z(\beta)}{\partial \beta}=k \ln \left[\frac{1}{2} e^{L \beta \rho^{2}+N \beta} \sqrt{M \beta}\left(\frac{2 \lambda e^{\left(M \beta / \lambda^{2}\right)}}{\sqrt{M \beta}}-2 \sqrt{\pi} \operatorname{erfi}\left(\frac{\sqrt{M \beta}}{\lambda}\right)-2 \sqrt{\pi}\right)\right] \\
- & \frac{1}{e^{L \beta \rho^{2}+N \beta} \sqrt{M \beta}\left[\frac{2 \lambda e^{\left(M \beta / \lambda^{2}\right)}}{\sqrt{M \beta}}-2 \sqrt{\pi} \operatorname{erfi}\left(\frac{\sqrt{M \beta}}{\lambda}\right)-2 \sqrt{\pi}\right]} \\
\times & {\left[2 k \beta \left[\frac{1}{2}\left(L \rho^{2}+N\right) e^{L \beta \rho^{2}+N \beta} \sqrt{M \beta}\left[\frac{2 \lambda e^{\left(M \beta / \lambda^{2}\right)}}{\sqrt{M \beta}}-2 \sqrt{\pi} \operatorname{erfi}\left(\frac{\sqrt{M \beta}}{\lambda}\right)-2 \sqrt{\pi}\right]\right.\right.}
\end{aligned}
$$


$\left.\left.+\frac{1}{4} \frac{e^{L \beta \rho^{2}+N \beta}\left[\frac{2 \lambda e^{\left(M \beta / \lambda^{2}\right)}}{\sqrt{M \beta}}-2 \sqrt{\pi} e r f i\left(\frac{\sqrt{M \beta}}{\lambda}\right)-2 \sqrt{\pi}\right] M}{\sqrt{M \beta}}-\frac{1}{2} \frac{e^{L \beta \rho^{2}+N \beta} \lambda e^{\left(M \beta / \lambda^{2}\right)}}{\beta}\right]\right]$

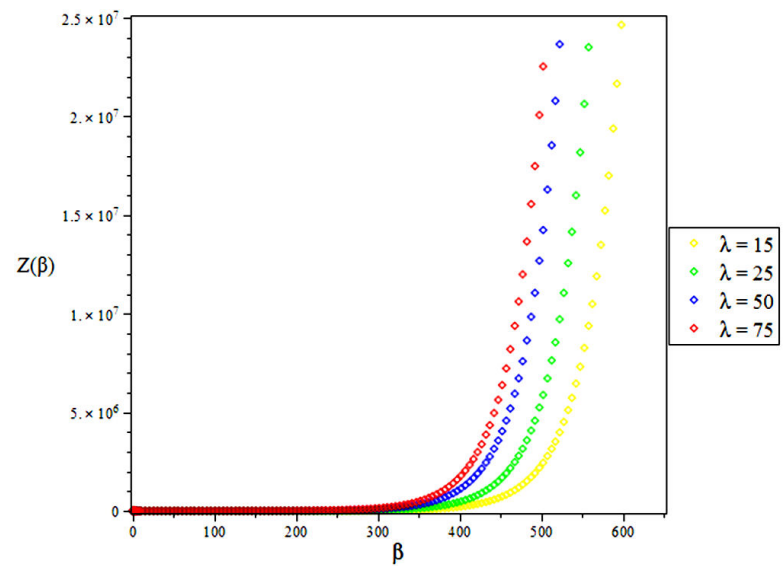

FIGURE 1. Vibrational Partition Function as a function of $\beta$ for different $\lambda$ (with $D=3$ ).

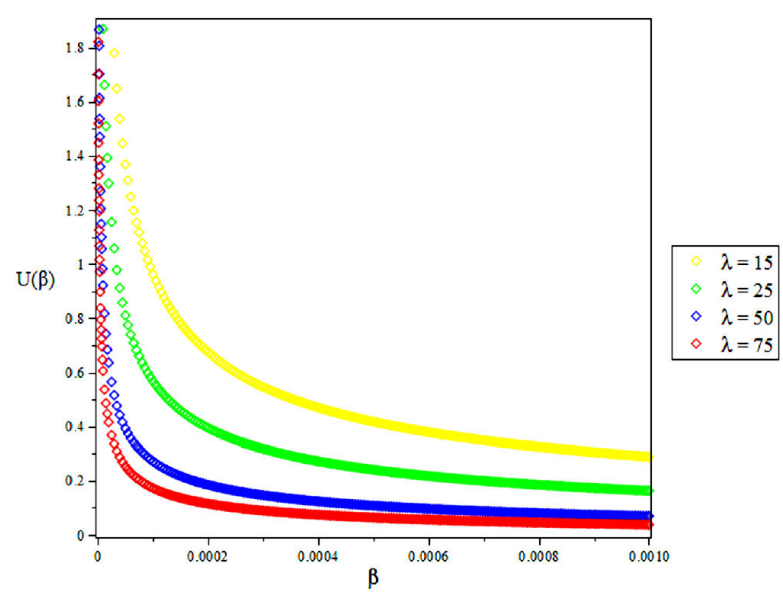

FIGURE 2. Vibrational Mean Energy as a function of $\beta$ for different $\lambda$ (with $D=3$ ).

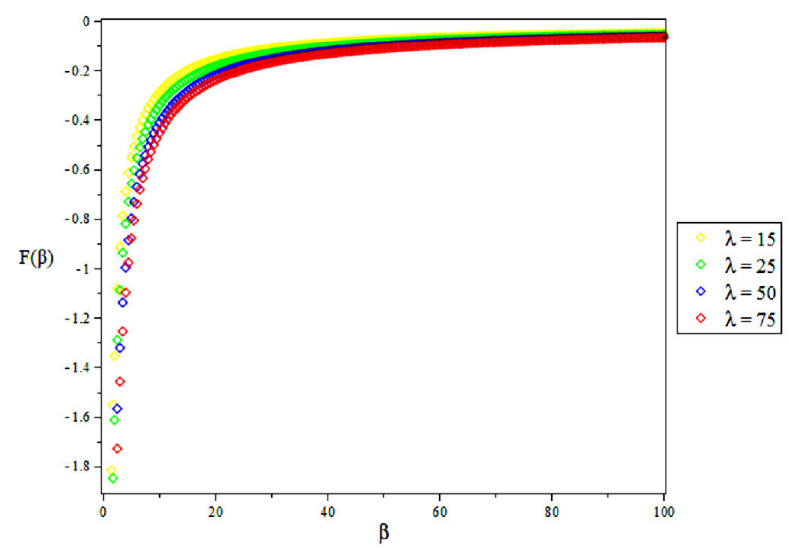

FIGURE 3. Vibrational Mean Free Energy as a function of $\beta$ for different $\lambda$ (with $D=3$ ).

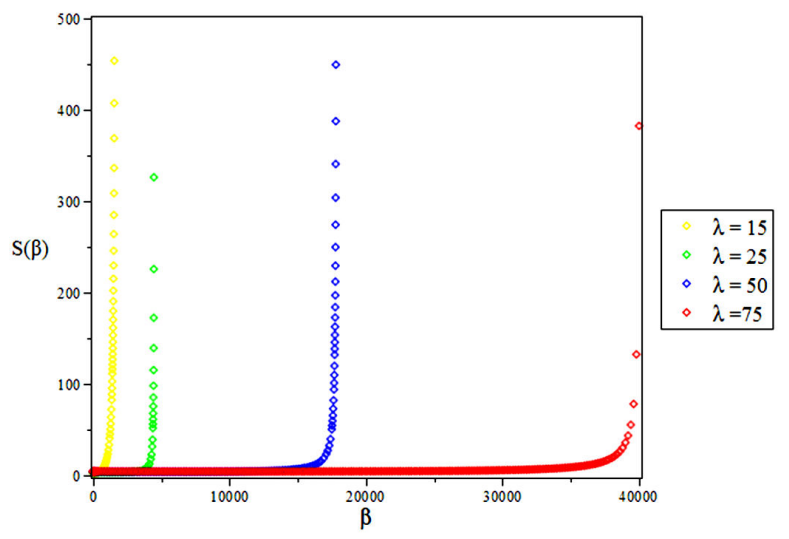

FIGURE 4. Vibrational Entropy as a function of $\beta$ for different $\lambda$ (with $D=3$ ).

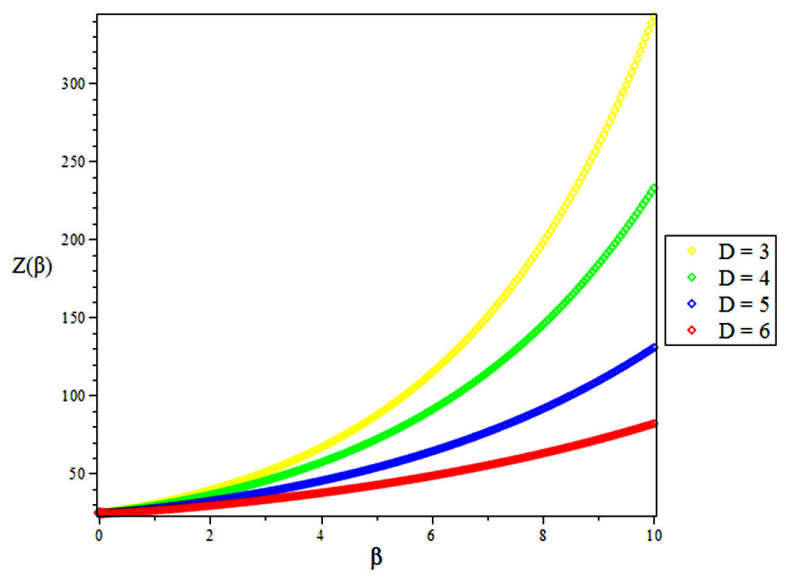

FIgURE 5. Vibrational Partition Function as a function of $\beta$ for different $D$ (with $\lambda=25$ ).

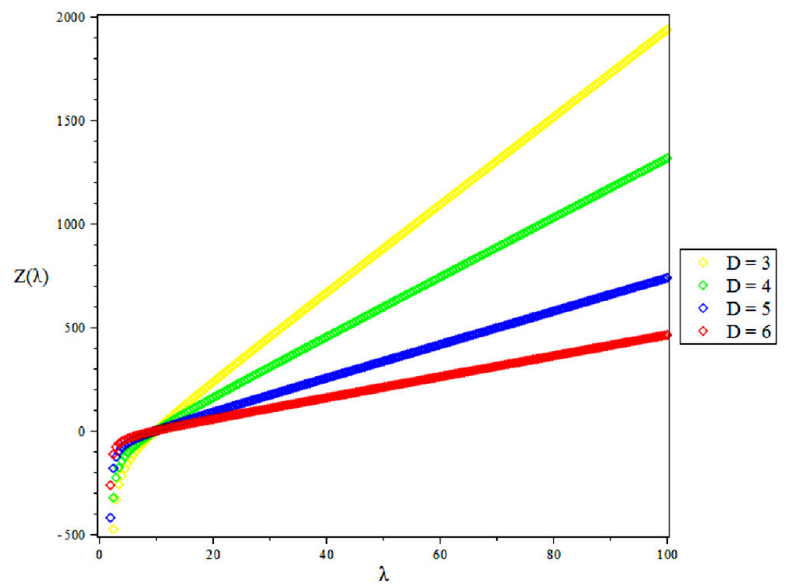

FIGURE 6. Vibrational Partition Function as a function of $\lambda$ for different $D$ (with $\beta=10$ ). 


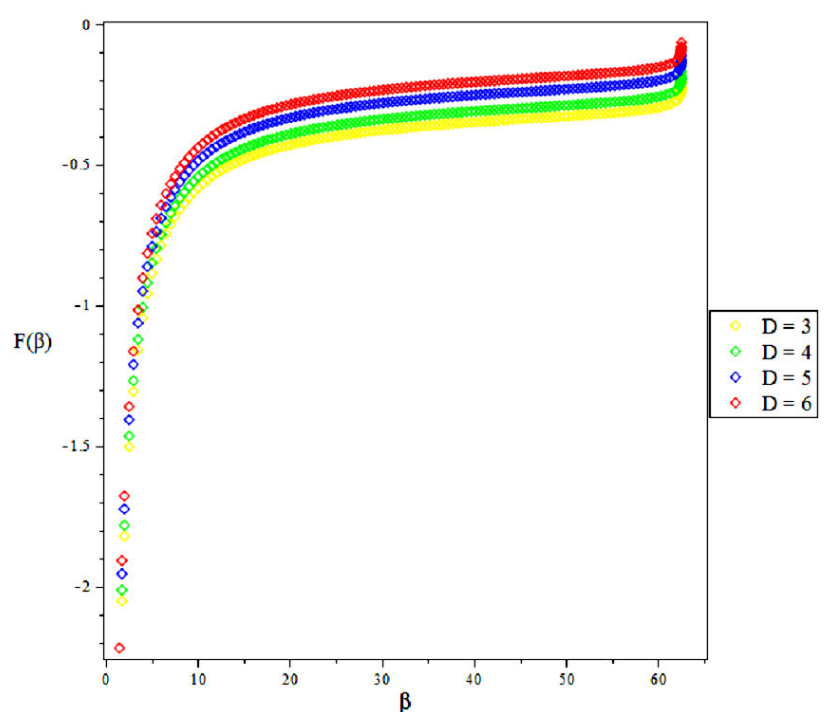

FIGURE 7. Vibrational Mean Free as a function of $\beta$ for different $D$ (with $\lambda=25$ ).

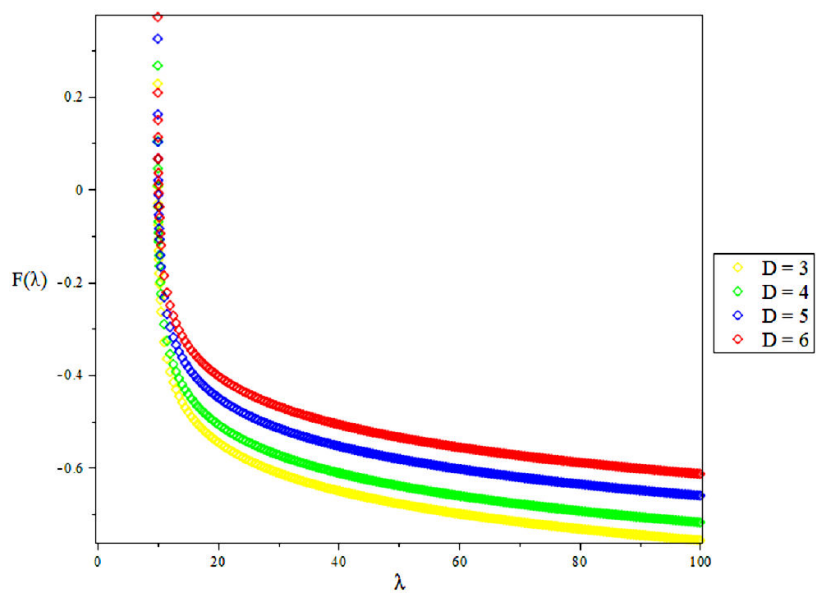

FIGURE 8. Vibrational Mean Free Energy as a function of $\lambda$ for different $D$ (with $\beta=10$ ).

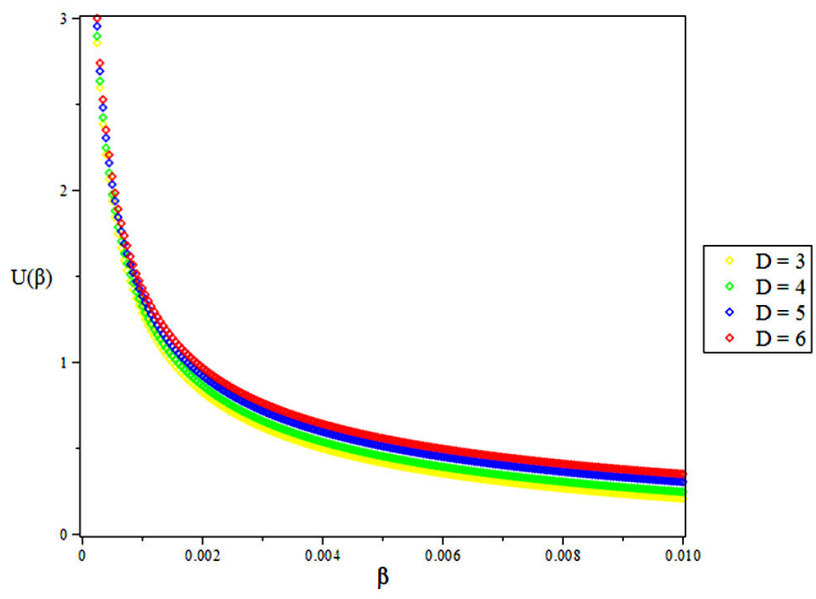

FIGURE 9. Vibrational Mean Energy as a function of $\beta$ for different $D$ (with $\lambda=25$ ).

\section{Results and Discussions}

In this research, the electronic state of Rubidium dimer $R b_{2}\left(X^{1} \sum_{g}^{+}\right)$molecule is considered. We take the experimental values of $\left(X^{1} \sum_{g}^{+}\right)$state of Rubidium $\left(R b_{2}\right)$ dimer from the literature [41]: $\alpha=0.055 \times 10^{3}\left(\mathrm{~cm}^{-1}\right)$ and $\mu=42.4559$ a.m.u. With the experimental data given above as inputs, the vibrational partion function and other thermodynamic functions are plotted for the $\left(X^{1} \sum_{g}^{+}\right)$state of $\left(R b_{2}\right)$ for various values of maximum vibration quantum number, $\lambda$, in Fig. 1-4, respectively. In Fig. 1, the partition function and $\beta$ increases with a decrease in maximum vibration quantum number. Figure 2 shows a monotonic decrease in vibrational mean energy as $\beta$ and $\lambda$ increases. We observe a monotonic increase in mean free energy and $\beta$ as $\lambda$ decreases in Fig. 3. In Fig. 4, there is a sharp increase in vibrational entropy at specific $\beta$ as the maximum vibration quantum number increases. It is worthy to note that the above figures are considered at a specific dimension $(D=3)$. Variations of the thermodynamic functions with temperature and maximum vibration quantum number were also considered for different dimensions. Figure 5 shows an increase in partition function and temperature as the dimension increase, at a maximum vibration quantum number of 25 . In Fig. 6, the partition function increases with increase in $\lambda$ from the origin, as $D$ increases with low temperatures $(\beta=10)$. A monotonic increase in mean free energy is observed in Fig. 7 as the temperature increase, with a decrease in dimension with a constant $\lambda=25$. Figure 8 shows a decrease in mean free energy with an increase in $\lambda$ as the dimension decreases at a constant $\beta=10$. In Fig. 9, there is a monotonic decrease in mean energy as $\beta$ increases with a decrease in dimension at constant $\lambda=25$. Figure 10 shows a slow monotonic decrease in vibrational entropy as maximum vibration quantum number increases with decrease in dimension at a constant $\beta=10$.

\section{Conclusion}

In the present study, the Schrödinger equation has been solved in D-dimension with QEP using the modified Factor-

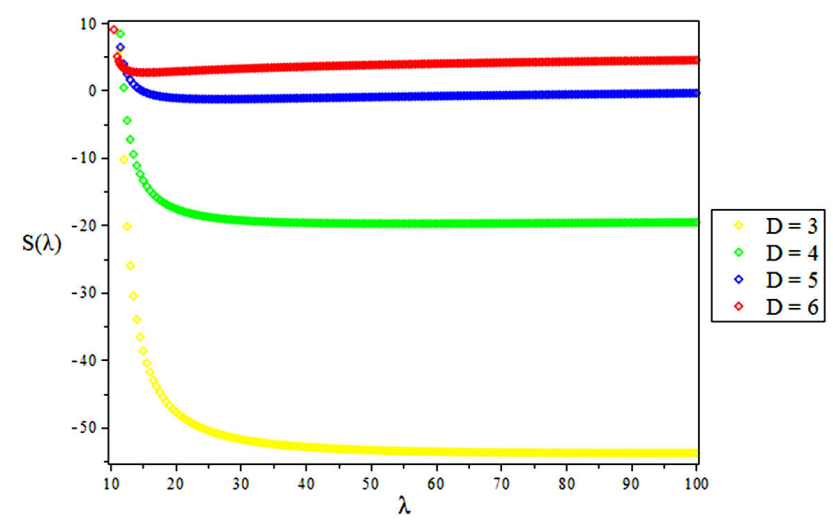

FIGURE 10. Vibrational Entropy as a function of $\lambda$ for different $D$ (with $\beta=10$ ). 
ization method and the energy eigenvalues and total wavefunctions obtained in terms of hypergeometric function. We have also calculated the partition function and other thermodynamic properties of vibrational mean energy. Vibrational mean free energy and vibrational entropy, for the electronic state of $\left(X^{1} \sum_{g}^{+}\right)$Rubidium $\left(R b_{2}\right)$ dimer. The behaviour of these thermodynamic functions with temperature and maximum vibration quantum numbers at specific dimension and at different dimensions, respectively have been plotted and discussed extensively. The QEP model has been noted to be most applicable in the study of molecular dynamics.
1. W. A. Yahya and K. J. Oyewumi, Journal of the Association of Arab Univ. for Basic and Appl. Sci. 1815 (2015).

2. A. N. Ikot, L. E. Akpabio and E. J. Uwah, Electr. J. Theor. Phys. 8 (2011) 225.

3. W. C. Qiang and S. H. Dong, Phys. Lett. A 368 (2007) 13.

4. H. Hassanabadi, B. H. Yazarloo, A. N. Ikot, N. Salehi and Z. Zarrinkamar, Ind. J. Phys. (2013).

5. C. S. Jia, X. L. Zeng, S. C. Li, L. T. Sun and Q. B. Yang, Commun. Theor. Phys. 37 (2002) 523.

6. A. N. Ikot, Chin. Phys. Lett. 29 (2012) 060307.

7. A. N. Ikot, O. A. Awoga and A. D. Antia, Chin. Phys. B 22 (2013) 020304.

8. B. I. Ita, A. I. Ikeuba, L. M. Hitler and P. Tchoua, J. Theor. Phys. Crypt. 10 (2015)

9. C. A. Onate and J. O. .A Idiodi, Chin. J. Phys. 53 (2015) 7.

10. A. D. Antia, A. N. Ikot, H. Hassanabadi and E. Maghsoodi, Ind. J. Phys. 87 (2013) 1133.

11. A. N. Ikot, O. A. Awoga, H. Hassanabadi and E. Maghsoodi, Comm. Theor. Phys. 61 (2014) 457.

12. B. J. Falaye, K. J. Oyewumi and M. Abbas, Chin. Phys. B 22 (2013) 110301.

13. C. A. Onate and J. O. Ojonubah, J. Theo. Appl. Phys. 10 (2016) 21.

14. A. N. Ikot, H. P. Obong, T. M. Abbey, S. Zare, M. Ghafourian and H. Hassanabadi, Few-Body Syst. 57 (2016) 807.

15. C. A. Onate, M. C. Onyeaju, A. N. Ikot and J. O. Ojonubah, Chin. J. Phys. 000 (2016) 1.

16. C. A. Onate, A. N. Ikot, M. C. Onyeaju and M. E. Udoh Karbala, Int. J. Modern Sci. 3 (2017) 1.

17. H. Ciftci, R. L. Hall and N. Saad, Phys. Lett. A 340 (2005) 288.

18. W. C. Qiang and S. H. Dong, Euro. Phys. Lett. 45 (2010) 10003.

19. S. M. Ikhdair and R. Sever, J. Math. Chem. 45 (2009) 1137.

20. S. H. Dong, Factorization method in Quantum Mechanics, (Springer, Armsterdam 2007).
21. C. S. Jia and Y. Jia, Eur. Phys. J. D. 71 (2017) 3.

22. B. J. Falaye, K. J. Oyewumi, S. M. Ikhdair and M. Hamzavi, Phys. Scr. 89 (2014) 115204.

23. H. M. Tang, G. C. Liang, L. H. Zhang, F. Zhao and C. S. Jia, Can. J. Chem. 92 (2014) 341.

24. J. Y. Liu, X. T. Hu and C. S. Jia, Can. J. Chem. 92 (2014) 40.

25. J. Y. Liu, G. U. Zhang and C. S. Jia, Phys. Lett. A 377 (2013) 1444.

26. X. Zou, L. Z. Yi and C. S. Jia, Phys. Lett. A 346 (2005).

27. M. Eshghi and M. Hamzavi, Commun. Theor. Phys. 57 (2012) 355.

28. A. Arda, R. Server and C. Tezcan, Chin. J. Phys. 48 (2010) 27.

29. A. N. Ikot, O. A. Awoga, H. Hassanabadi and E. Maghsoodi, Commun. Theor. Phys. 61 (2014) 457.

30. K. J. Oyewumi, B. J. Falaye, C. A. Onate, O. J. Oluwadare and W. A. Yahya, Mol. Phys. 112 (2014) 127.

31. S. H. Dong, M Lozada-Cassou, J. Yu, F. J. Angeles and A. L. Rivera, Int. J. Quant. Chem. 107 (2007) 366.

32. S. M. Ikhdair and B. J. Falaye, Chem. Phys. 421 (2013) 82.

33. S. H. Dong and M. Cruz-Irisson, J. Math. Chem. 50 (2012) 881.

34. A. N. Ikot, B. C. Lutfuoglu, M. I. Ngweke, M. E. Udoh, S. Zare and H. Hassanabadi, Euro. Phys. J. Plus, 131 (2016) 419.

35. X. Q. Song, C. W. Zhang and C. S. Jia, Chem. Phys. Lett. 673 (2017) 50

36. C. S. Jia, L. H. Zhang and C. W. Wang, Chem. Phys. Lett. 667 (2017) 211.

37. H. Hassanabadi, B. Y. Yazarloo, S. S. Zarrinkamar and H. Rahimov, Commun. Theor. Phys. 57 (2012) 339.

38. S. H. Dong, Commun. Theor. Phys. 55 (2011) 969.

39. C. S. Jia, C. W. Wang, L. H. Zhang, X. L. Peng, R. Zeng and X. T. You, Chem. Phys. Lett. 676 (2017) 150.

40. C. S. Jia, L. H. Zhang and C. W. Wang, Chem. Phys. Lett. 667 (2017) 211.

41. S. Kaur and C. G. Mahajan, Pramana-J. Phys. 52 (1999) 0459 